\title{
Effects of Commercial Nitrilase Hydrolysis on Acrylic Fabrics
}

\author{
Hye Rim $\mathrm{Kim}^{\dagger}$ and Hye Young Seo \\ Dept. of Clothing and Textiles, Sookmyung Women's University; Seoul, Korea
}

\begin{abstract}
This study aims to evaluate the hydrolytic activity of a commercial nitrilase and optimize nitrilase treatment conditions to apply eco-friendly finishing on acrylic fabrics. To assess the possibility of hydrolyzing nitrile bonds in acrylic fabric using a commercial nitrilase, the amounts of hydrolysis products, ammonia and carboxylate ions, were measured. The treatment conditions were optimized via the amount of ammonia. The formation of carboxylate ions on the fabric surface was detected by X-ray photoelectron spectroscopy and wettability measurements. After nitrilase treatment, ammonia was detected in the treatment liquid; thus, nitrilase hydrolyzed the nitrile bonds in acrylic woven fabric. The largest amount of ammonia was released into the treatment liquid under the following conditions: $\mathrm{pH} 8.0,40^{\circ} \mathrm{C}$, and a treatment time of $5 \mathrm{~h}$. The formation of carboxylate ions on the acrylic woven fabric surface by nitrilase hydrolysis was proven by the increased O1s content measuring of XPS analysis. From comparison of the results of nitrilase and alkaline hydrolysis, the white index and strength of the alkali-hydrolyzed acrylic fabric decreased, whereas those of the nitrilase-hydrolyzed samples were maintained. The nitrilase hydrolysis improved the sensitivity of acrylic fabrics to basic dye similarly to alkaline hydrolysis without the drawbacks of yellowing and decreased strength caused by alkaline hydrolysis.
\end{abstract}

Key words : nitrilase, acrylic, enzyme, eco-friendly, hydrolytic activity

\section{Introduction}

Surface modification is considered to be a valuable tool for improving the quality of fibers (Battistel et al., 2001). Conventional methods for modifying synthetic polymers, acrylic fibers, use strong chemicals at high temperatures. By the alkaline hydrolysis (e.g., $\mathrm{NaOH}$ ), the nitrile groups (-CN-) in acrylic fibers converted into carboxylate ions (COONa) on fabrics and ammonia $\left(\mathrm{NH}_{4} \mathrm{OH}\right)$, thus on the surface of fabrics carboxylate ionic groups were formed (Battistel et al., 2001; Bornscheuer \& Kazlauskas, 2005; Gübitz \& Cavaco-Paulo, 2003; Kobayashi \& Shimizu, 2000; Matamá, et al., 2007).

Acrylic fibers hydrolyzed respect to the environmental impact, however, biotechnologies, such as enzymatic catalysis-based techniques, are required to modify acrylic fibers with a reduced need for harsh chemicals (Battistel et al., 2001). There are two different pathways for the enzymatic modification of polyacrylonitrile (acrylic) fibers: One method uses nitrile hydratases (NHase, EC 4.2.1.84; lyase), and the other uses nitrilases (EC 3.5.5.1) (Babu et al., 2011; Bornscheuer \& Kazlauskas, 2005; Kobayashi \& Shi-

†Corresponding author; Hye Rim Kim

Tel. +82-2-2077-7591, Fax. +82-2-2077-7324

E-mail: khyerim@sm.ac.kr

(C) 2016 (by) the authors. This article is an open access article distributed under the terms and conditions of the Creative Commons Attribution license (http://creativecommons.org/licenses/by/3.0/), which permits unrestricted use, distribution, and reproduction in any medium, provided the original work is properly cited. mizu, 2000). NHase hydrolysis involves two enzymes: NHase, which catalyzes the hydrolysis of a nitrile to an amide and amidase (EC 3.5.1.4), which sequentially converts the amide to carboxylate ions and ammonia (Battistel et al., 2001; Kobayashi \& Shimizu, 2000; Tauber et al., 2000). In the other pathway, nitrilase (EC 3.5.5.1) directly hydrolyzes the nitrile to produce ammonia and the corresponding carboxylate ions on the fabric surface.

Only a few studies have investigated the effects of nitrilase hydrolysis on acrylic fibers (Fischer-Colbrie et al., 2007; Matamá et al., 2007). Fisher-Colbrie et al. (2007) was provided limited data on the effects of nitrilase cultured in vitro on acrylic fibers or powder. Even though commercial nitrilase and fabrics have been used as experimental specimens in the study by Matamá et al. (2007), the optimized conditions have not been elucidated. In order to apply nitrilase hydrolysis for acrylic fabric-finishing processes, the optimum nitrilase conditions should be determined to facilitate ecofriendly finishing in the acrylic fabric industry. Moreover, no study to date has compared whether nitrilase hydrolysis has similar reactivity or drawbacks as alkaline hydrolysis.

Therefore, this study aims to evaluate the effects of commercial nitrilase on acrylic woven fabrics to further environmentally friendly finishing. To achieve this goal, the following are necessary: (1) Use of a commercial nitrilase, (2) determination of the optimum treatment conditions using a commercial nitrilase, and (3) comparison of the effects of nitrilase and alkaline hydrolysis on the properties of the fabrics. If commercial nitrilase can hydrolyze acrylic fabrics, the carboxylate ions $\left(\mathrm{COO}^{-}\right)$can be formed on fab- 
ric surfaces and ammonia $\left(\mathrm{NH}_{4}{ }^{+}\right)$is going to be produced as hydrolysis products in treatment liquid. Thus, the present study aims to optimize the commercial nitrilase hydrolysis conditions by measuring the production of ammonia, which is a hydrolysis product. In addition, the formation of carboxylate ions on the surfaces of the acrylic woven fabrics by nitrilase is evaluated by X-ray photoelectron spectroscopy and wettability measurements. In order to evaluate whether nitrilase hydrolysis has similar reactivity or drawbacks with alkaline hydrolysis, the fabric properties, i.e., sensitivity to basic dye, whiteness, strength, and weight loss, after alkaline and nitrilase hydrolysis are compared.

\section{Experimental}

\subsection{Materials}

All experiments were conducted using acrylic woven fabric (Table 1). All fabric samples were washed with $2 \mathrm{~g} / \mathrm{L}$ Triton X-100 at $50^{\circ} \mathrm{C}$ for $30 \mathrm{~min}$, and then rinsed with water at $50^{\circ} \mathrm{C}$ for $30 \mathrm{~min}$ to desize. Commercially available nitrilase was used without further purification (Table 2). The following chemicals were used without further purification: sodium phosphate $\left(\mathrm{p} K_{\mathrm{a}}\right.$ 7.2, Sigma Chemical Co., USA), Triton X-100 (nonionic surfactant; Sigma Chemicals Co., USA), sodium carbonate (Duksan Pure Chemicals, Korea), sodium hydroxide (Duksan Pure Chemicals, Korea), acetic acid (Duksan Pure Chemicals, Korea), rochelle salt (aqueous solution: $50 \%$ potassium sodium tartrate tetrahydrate, Sigma Chemical Co., USA), Nessler reagent (Sigma Chemical Co., USA), ammonium chloride $\left(\mathrm{NH}_{4} \mathrm{Cl}\right.$, Duksan Pure Chemicals, Korea), and basic dye (methylene blue, C.I. basic blue 9, Duksan Pure Chemicals, Korea).

\subsection{Enzymatic hydrolysis}

Fig. 1 shows a schematic of the nitrilase hydrolysis reaction performed to optimize the treatment conditions; different $\mathrm{pH}$ values, temperatures, and treatment times on acrylic woven fabrics were investigated.

Table 1. Fabric characteristics

\begin{tabular}{ccccc}
\hline Fiber (\%) & Weave & $\begin{array}{c}\text { Fabric count } \\
(\text { yarns/inch) }\end{array}$ & $\begin{array}{c}\text { Weight } \\
\left(\mathrm{g} / \mathrm{m}^{2}\right)\end{array}$ & $\begin{array}{c}\text { Weight } \\
\left(\mathrm{g} / \mathrm{m}^{2}\right)\end{array}$ \\
\hline Acrylic 100 & Plain & $88 \times 80$ & 110 & 0.273 \\
\hline
\end{tabular}

Table 2. Enzyme characteristics

\begin{tabular}{cccc}
\hline Enzymes & Source & Activity & Manufacturer \\
\hline Nitrilase & Recombinant, & & Sigma Chemicals \\
(EC 3.5.5.1) & expressed in & $\geq 2.0 \mathrm{U} / \mathrm{mg}$ & Co. USA \\
& E. coli & & \\
\hline
\end{tabular}

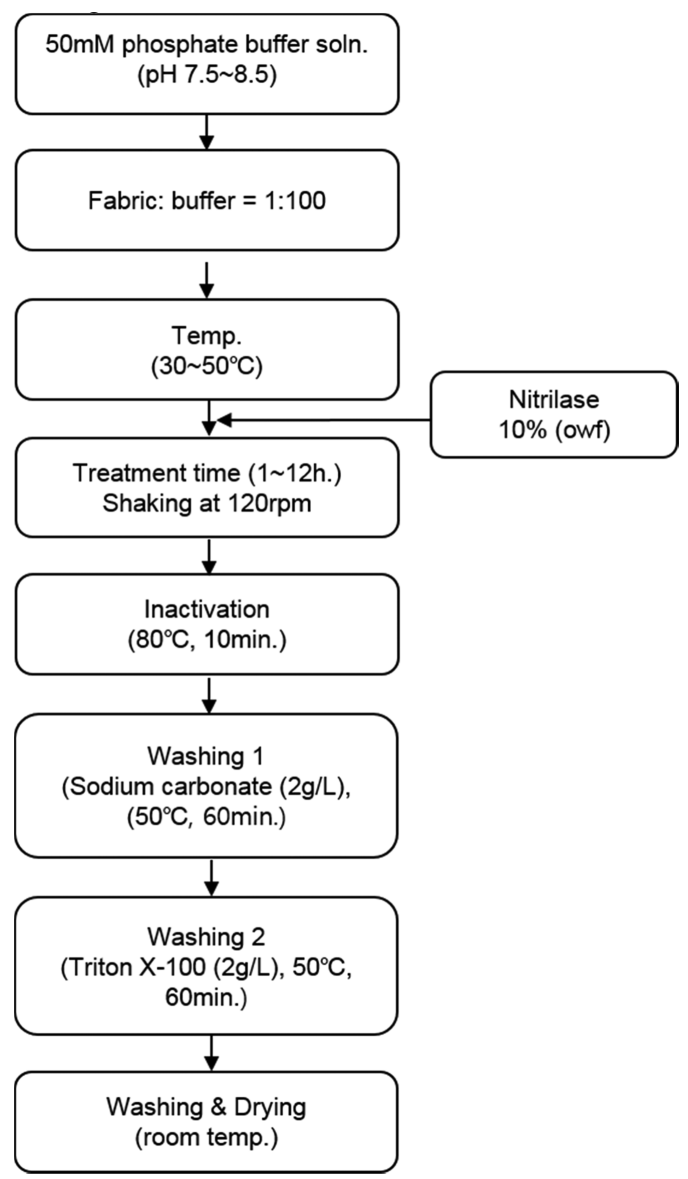

Fig. 1. The nitrilase treatment process on acrylic fabric.

2.3. Optimization of treatment conditions by evaluating nitrilase hydrolytic activity

Ammonia estimation is a practical method for determining nitrilase activity (Banerjee et al., 2003). The amount of ammonia released into the treatment liquid was quantified using the Nessler method (Matamá et al., 2007). The Nessler method performed as the following steps (Fischer-Colbrie et al., 2007; Matamá et al., 2007): After nitrilase hydrolysis, $0.5 \mathrm{~mL}$ of the treatment liquid was diluted with $4.5 \mathrm{~mL}$ distilled water in glass tubes, and $10 \mu \mathrm{L}$ of Rochelle salt solution was added to the mixture. After mixing well, $2 \mathrm{~mL}$ of Nessler's reagent was added to each tube. The color was allowed to develop for $10 \mathrm{~min}$ and the absorbance was recorded at $390 \mathrm{~nm}$ using a UV-vis spectrometer (S-3100; Scinco Co., Korea). A calibration curve, constructed by using a standard solution of ammonium chloride.

\subsection{The effects of nitrilase hydrolysis on the fabric}

When nitrilase hydrolyzes nitrile bonds, corresponding carboxylate ions groups form on the fabric surfaces. Fabric surface analysis after nitrilase hydrolysis was carried out using X-ray 
photoelectron spectroscopy (XPS). The XPS signal is specific to a polymer surface, as the signals result from a combination of interactions with surfaces and layers immediately underneath (Babu et al., 2011; Tauber et al., 2000). Changes in acrylic fabric surface by nitrilase hydrolysis was analyzed with an AXIS Nova-XRF (WD) (Kratos Analytical Co., UK).

The change of wettability of the nitrilase-treated acrylic fabric was evaluated through its water absorbency and water contact angle (WCA). The test of water absorbency was evaluated according to AATCC 79-1992. The WCA value was determined using a contact-angle measurement system (KRÜSS DSA 100; KRÜSS Inc., Germany). The sample was dosed with $3 \mu \mathrm{L}$ of water in each test, and the results of each test were obtained from the first value after dosing. Each test was conducted ten times.

\subsection{Comparison of nitrilase and alkaline hydrolysis}

Alkaline-treated samples were prepared to compare the properties of nitrilase- and alkaline-treated acrylic fabrics. Alkaline hydrolysis of acrylic fabrics was performed using sodium hydroxide $(\mathrm{NaOH})$. The treatment method was modified from those of other researchers (Gupta et al., 2004; Kim et al., 2002). The acrylic fabrics were hydrolyzed as follows: $1 \mathrm{~N} \mathrm{NaOH}$ at a liquor ratio of 100:1 ( $\mathrm{NaOH}$ solution/fabric) at $90^{\circ} \mathrm{C}$ for $3 \mathrm{~h}$. After alkaline hydrolysis, neutralization was performed using acetic acid. The acrylic fabrics were then washed with $2 \mathrm{~g} / \mathrm{L}$ acetic acid solution at a liquor ratio of 100:1 (acetic acid solution/fabric) at $60^{\circ} \mathrm{C}$ for $30 \mathrm{~min}$. The samples were rinsed thoroughly using tap water and dried at room temperature.

Nitrilase- and alkaline-hydrolysis in acrylic fabrics were compared by the sensitivity to basic dye, whiteness, weight loss, and tensile strength. The measurement of sensitivity to basic dye was simple way to detect the change of the amount of carboxylate ions on the surface of acrylics (Babu et al., 2011; Gupta et al., 2004; $\mathrm{Kim}$ et al., 2002). Acrylic fabrics was stained at $80^{\circ} \mathrm{C}$ for $60 \mathrm{~min}$ with basic dye ( $0.5 \%$ owf, C.I. basic blue 9$)$ at a liquor ratio of 50:1. The $\mathrm{pH}$ of the basic dye solution (without $\mathrm{pH}$ adjustment) was around 7.0. After staining, the samples were washed with Triton X-100 ( $2 \%$ owf $)$ at $50^{\circ} \mathrm{C}$ and then rinsed with tap water. The sensitivity to basic dye was evaluated by UV-vis spectrophotometer. The absorbance (ABS) of the staining liquid at $670 \mathrm{~nm}$ was recorded before and after staining using a UV-vis spectrophotometer. Each test was conducted five times. The sensitivity was calculated using the following equation:

Sensitivity to basic dye (\%)

$$
=\frac{\mathrm{ABS}_{\text {before staining }}-\mathrm{ABS}_{\text {after staining }}}{\mathrm{ABS}_{\text {before staining }}} \times 100
$$

Where $\mathrm{ABS}_{\text {before staining }}$ and $\mathrm{ABS}_{\text {after staining }}$ are the absorbance at $670 \mathrm{~nm}$ of the dye solution before and after the dyeing process, respectively.

The white index was measured using a computational color matching system (JX-777, Japan). The white index was calculated as follows:

$$
\mathrm{WI}(\mathrm{Lab})=100-\left[(100-\mathrm{L})^{2}+\mathrm{a}^{2}+\mathrm{b}^{2}\right]^{1 / 2}
$$

The tensile strength was determined by the strip method in accordance with KS K 0521. The tensile strength loss was calculated using the following equation:

Tensile strength loss $(\%)=\frac{\mathrm{S} 1-\mathrm{S} 2}{\mathrm{~S} 1} \times 100$

$\mathrm{S}_{1}$ : Tensile strength of untreated fabric

$\mathrm{S}_{2}$ : Tensile strength of treated fabric

The weight loss was evaluated from the dry weight of the fabric before and after alkaline or nitrilase hydrolysis.

For the statistical comparison between nitrilase and alkaline hydrolysis, the SPSS 20 software package was utilized. Statistically significant factors of the sensitivity to basic dye, whiteness, and tensile strengths were determined by analysis of variance (one-way ANOVA). Multiple comparison tests using the Duncan test were conducted as post hoc tests. The factors were considered as significant at $p$ values of less than 0.05 .

Changes in the surface of the acrylic fabrics were analyzed by scanning electron microscopy (SEM, S-3500N, Hitachi, Japan) after plating the samples with platinum.

\section{Results \& Discussion}

\subsection{Optimization of treatment conditions via nitrilase} hydrolytic activity

When nitrilase hydrolyzes nitriles in acrylic fabric, ammonia is released into the treatment liquid and carboxylate ions are formed on the fabric surface. Fig. 2 shows the effects of treatment time on the amount of ammonia released into the treatment liquid. The acrylic fabrics were hydrolyzed for $1 \sim 12 \mathrm{~h}$ with nitrilase $(10 \%$ owf) at $\mathrm{pH} 8.0$ and $40^{\circ} \mathrm{C}$. Hydrolysis of acrylic for $1 \mathrm{~h}$ resulted in a negligible amount of released ammonia. As the treatment time increased, the amount of ammonia increased gradually. The greatest amount of ammonia was obtained after $5 \mathrm{~h}$ of treatment time. The increase in the amount of ammonia released into the treatment liquid after nitrilase hydrolysis confirmed that nitrilase hydrolyzed the nitrile bonds in the acrylic fabrics (Matamá et al., 2007). The nitrilase from $E$. coli in this study hydrolyzed nitrile bonds in 


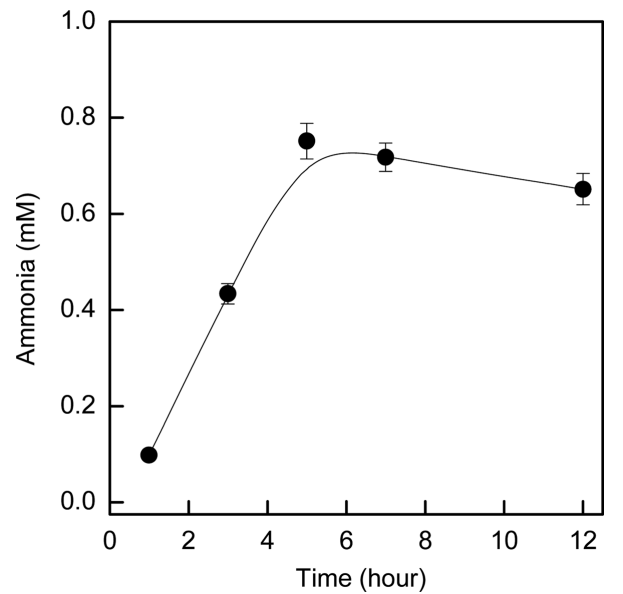

Fig. 2. Effects of treatment time on the hydrolytic activity of nitrilase. Treatment conditions: $10 \%$ (owf) nitrilase, $\mathrm{pH} 8.0,40^{\circ} \mathrm{C}$.

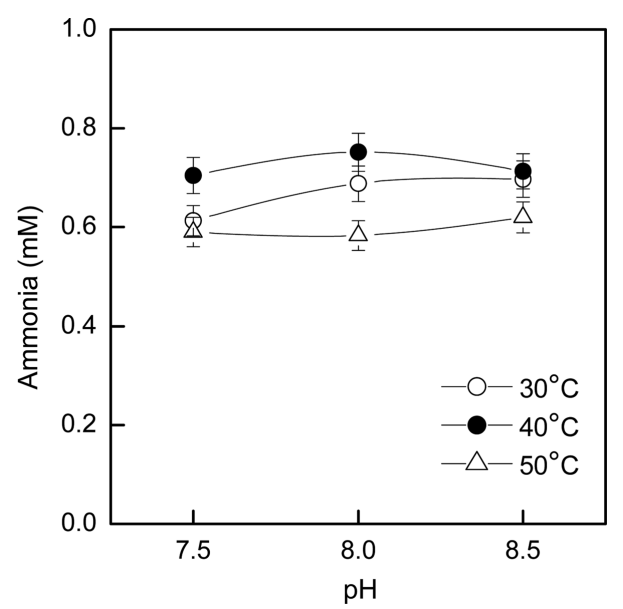

Fig. 3. Effects of $\mathrm{pH}$ and temperature on the hydrolytic activity of nitrilase. Treatment conditions: $10 \%$ (owf) nitrilase, 5 -h treatment time.

acrylic fabrics within a shorter time period compared the treatment time of $8 \mathrm{~h}$ in other study by Matamá et al. (2007). When nitrilase hydrolysis was performed above $5 \mathrm{hr}$, the amount of ammonia released into the liquid was decreased because a longer treatment time results in denaturation of the enzymes or aggregation of enzyme molecules (Gübitz \& Cavaco-Paulo, 2003; Kim \& Song, 2010).

Fig. 3 shows the effects of $\mathrm{pH}$ and temperature on nitrilase hydrolytic activity. When the acrylic fabrics were treated at $40^{\circ} \mathrm{C}$, a significant increase was observed in the amount of ammonia released as compared with the results at either $30^{\circ} \mathrm{C}$ or $50^{\circ} \mathrm{C}$. When the acrylic fabrics were treated at $50^{\circ} \mathrm{C}$, the hydrolytic activity of nitrilase was lower than that observed under the other conditions. The optimum temperatures of most nitrilases range from $30^{\circ} \mathrm{C}$ to $55^{\circ} \mathrm{C}$ (Gong et al., 2012), and the nitrilase in the present study effi- ciently hydrolyzed acrylic fabrics at $40^{\circ} \mathrm{C}$. Moreover, for alkaline hydrolysis, the acrylic fabrics were treated at relatively high temperatures $\left(\sim 80-95^{\circ} \mathrm{C}\right)$. Nitrilase hydrolysis, however, was performed at relatively mild conditions including a low temperature $\left(40^{\circ} \mathrm{C}\right)$ and $\mathrm{pH}$ value (8.0).

On the basis of the amount of ammonia released into the treatment liquid by nitrilase hydrolysis, the optimum conditions were determined to be $\mathrm{pH} 8.0$ at $40^{\circ} \mathrm{C}$ for a treatment time of $5 \mathrm{~h}$.

\subsection{Effects of nitrilase hydrolysis on the fabric surfaces}

To evaluate the change in the fabric surface by nitrilase hydrolysis, XPS spectra of untreated (a) and nitrilase-treated (b) acrylic fabrics were compared (Fig. 4 and Table 3). The oxygen peak of the treated sample is more intense than that of the untreated sample. The details of the XPS peak corresponding to the carbon atom (C1s) are shown in Fig. 4. The carbon peak of the nitrilase-treated sample is less intense than that of the untreated sample. The $\mathrm{C} 1 \mathrm{~s}$ peak also broadened from 281 to $288 \mathrm{eV}$, indicating that nitrogen was involved in the formation of chemical bonds with carbon in

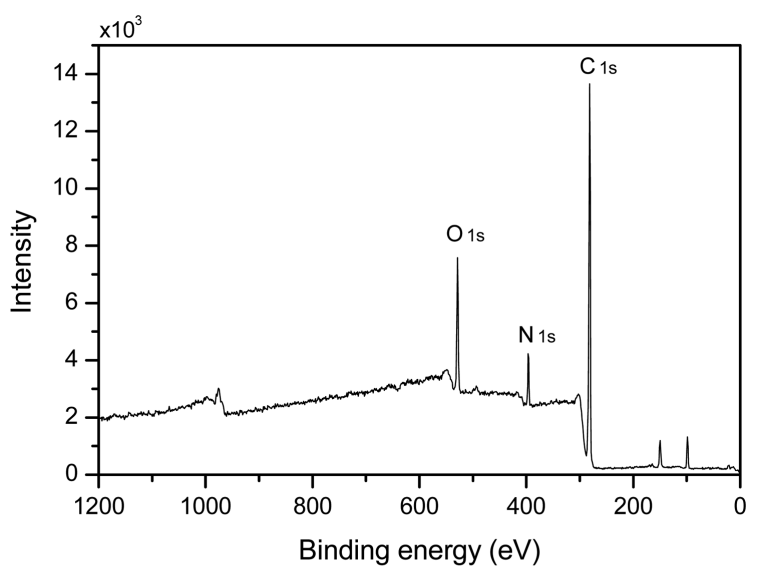

(a)

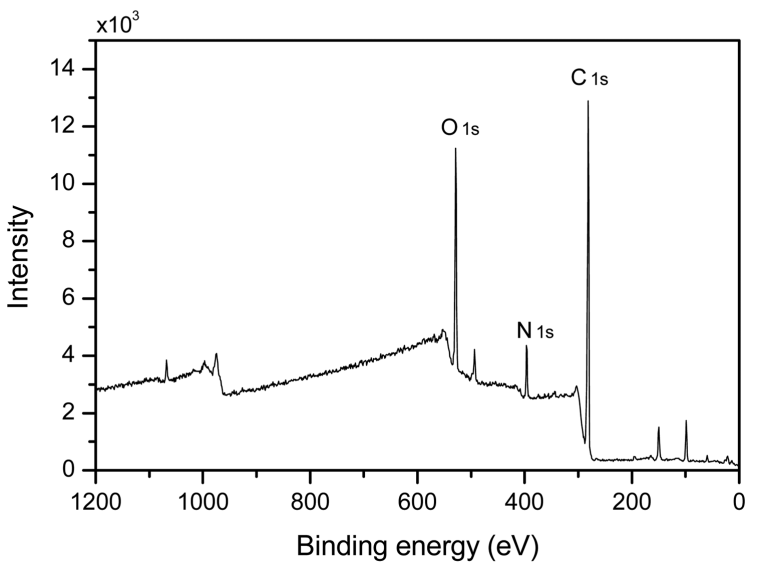

(b)

Fig. 4. XPS spectra of untreated (a) and nitrilase-treated (b) acrylic fabrics. 
Table 3. Atomic concentration on acrylic fabric surface by nitrilase treatment, as determined by XPS analysis

\begin{tabular}{cccc}
\hline Sample & Element & $\begin{array}{c}\text { Peak position } \\
(\mathrm{eV})\end{array}$ & $\begin{array}{c}\text { Atomic } \\
\text { concentration (\%) }\end{array}$ \\
\hline \multirow{4}{*}{ Untreated } & C 1s & 281.90 & 79.69 \\
& N 1s & 396.55 & 5.56 \\
& O 1s & 529.05 & 10.06 \\
\hline \multirow{3}{*}{ Nitrilase-treated } & C 1s & 281.85 & 71.51 \\
& N 1s & 396.70 & 5.23 \\
& O 1s & 529.05 & 16.34 \\
\hline
\end{tabular}

three possible chemical states, i.e., $\mathrm{C}-\mathrm{N}, \mathrm{C}=\mathrm{N}$, and $\mathrm{C} \equiv \mathrm{N}$ bonds (Majumdar et al., 2012). The differences between the $\mathrm{C}-\mathrm{N}$ and $\mathrm{C} \equiv \mathrm{N}$ bonds are not clear from analysis of the C1s XPS spectra because of the wide spectral ranges (Blyth et al., 2000; Majumdar et al., 2012; Wang et al., 2004). The content of C1s of the acrylic fabric decreased after nitrilase treatment (Table 3). Thus, we attributed the $\mathrm{C} 1 \mathrm{~s}$ peak $(281.9 \mathrm{eV})$ to the nitrile group $(\mathrm{C} \equiv \mathrm{N})$ in acrylic. Because nitrilase hydrolyzed the $\mathrm{CN}$ groups, the $\mathrm{C}-\mathrm{O}$ and $\mathrm{C}=\mathrm{O}$ components of the acrylic fabric increased accordingly. Thus, the $\mathrm{O} 1 \mathrm{~s}$ content increased because of the conversion of the nitrile bonds, as shown in Table 3. The insignificant variation of the nitrogen content was caused by the formation of amides as a side-product of nitrilase hydrolysis. The amount of amide varied with the structures of nitrile and nitrilase (Bornscheuer \& Kazlauskas, 2005).

The newly generated carboxylate ions on the fabric surface influenced the wettability of the fabrics (Battistel, 2001; Kim \& Song, 2010; Wang et al., 2004). Fig. 5 shows the values of WCA and water absorbency time of the fabrics after treatment under various conditions. The WCA value and water absorbency time of nitrilase-treated acrylic fabrics were lower than those of the untreated and buffer-treated samples. The presence of the hydro-

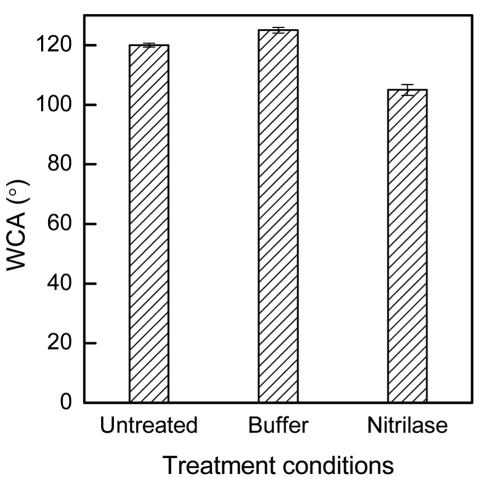

(a)

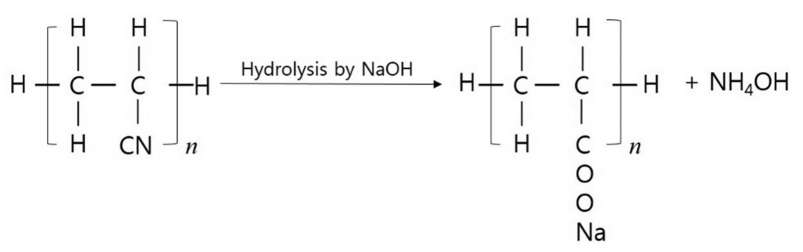

Fig. 6. Schematics of alkaline hydrolysis on acrylic fibers.

philic ionic groups, i.e., carboxylate ions, as proven via XPS, decreased the WCA value and water absorbency time. Therefore, the acrylic fabric surface became less hydrophobic with improved wettability.

\subsection{Comparison of nitrilase and alkaline hydrolysis}

Alkaline hydrolysis is a conventional process to hydrolysis of acrylic fabrics. By the alkaline hydrolysis, the nitrile groups in acrylic fibers converted into carboxylate ions on fabrics and ammonia (Fig. 6).

From the XPS and wettability measurement, the nitrilase hydrolysis was proved to form the carboxylate ionic groups on acrylic fabrics' surfaces. In order to compare the nitrilase hydrolysis with an alkaline hydrolysis, the reactivity of carboxylate ionic groups formed on fiber surfaces was compared by the sensitivity of basic dye. The measurement of sensitivity to basic dye was simple way to detect the change of the amount of carboxylate ions on the surface of acrylics (Babu et al., 2011; Gupta et al., 2004; Kim et al., 2002).

Fig. 7 shows the sensitivity of the fabric to basic dye after treatments under various conditions. The sensitivity of acrylic fabrics to basic dye increased after both nitrilase and alkaline hydrolysis because carboxylate ions were formed on the surface of acrylic fabrics upon nitrilase or alkaline hydrolysis (Fischer-Colbrie et al., 2007; Gupta et al., 2004; Kim et al., 2002; Matamá, et al., 2007).

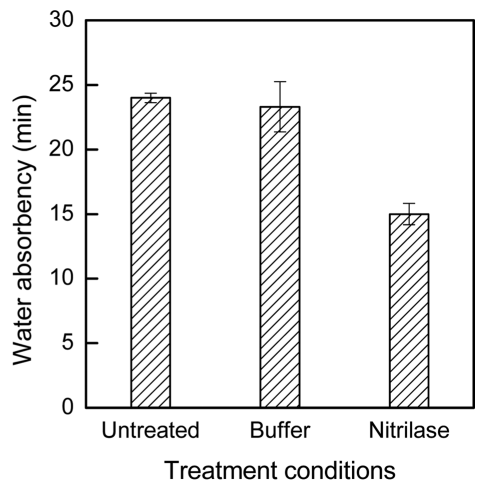

(b)

Fig. 5. WCA (a) and water absorbency (b) of acrylic fabrics after various treatment conditions. Buffer: buffer treatment at $\mathrm{pH} 8.0,40^{\circ} \mathrm{C}, 5-\mathrm{h}$ treatment time; Nitrilase: nitrilase treatment at $10 \%$ (owf) nitrilase, $\mathrm{pH} 8.0,40^{\circ} \mathrm{C}, 5$-h treatment time. 


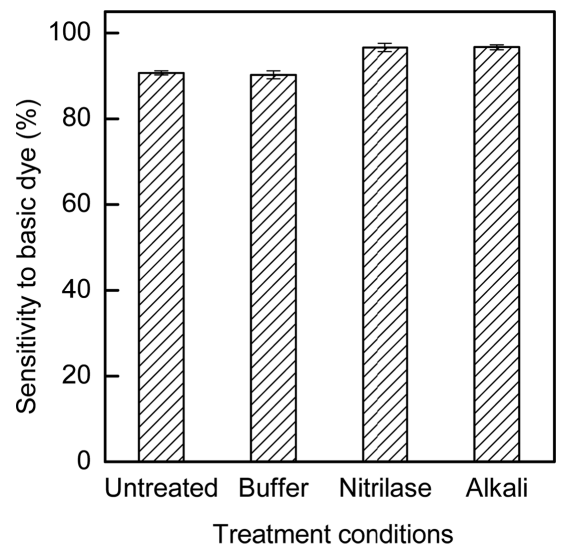

Fig. 7. Sensitivity of acrylic fabrics to basic dye after various treatment conditions. Buffer: buffer treatment at $\mathrm{pH} 8.0,40^{\circ} \mathrm{C}, 5$-h treatment time; nitrilase: Nitrilase treatment at $10 \%$ (owf) nitrile, $\mathrm{pH} 8.0,40^{\circ} \mathrm{C}$, 5-h treatment time. Alkali: alkaline treatment with $1 \mathrm{~N} \mathrm{NaOH}, 90^{\circ} \mathrm{C}, 3-\mathrm{h}$ treatment time.

To determine whether the influence of the treatment conditions had statistical significance, the sensitivity to basic dye was analyzed using SPSS. According to the Duncan analysis, the sensitivity to basic dye could be divided into the following two groups: (1) Untreated and buffer-treated samples and (2) nitrilase- and alkaline-treated samples. ANOVA of the sensitivity to basic dye was confirmed by the significance levels; in particular, $F=88.618$ and $p$ $=.000$ at $95 \%$. Thus, ANOVA demonstrated that nitrilase and alkaline hydrolysis had a statistically significant influence on the increase in sensitivity of the acrylic fabrics to basic dye. Moreover, the effects of nitrilase and alkaline hydrolysis were not significantly different. The nitrilase hydrolysis was proved to increase the sensitivity of the fabric to basic dye similarly to alkaline hydrolysis.

The drawbacks of alkaline hydrolysis of acrylics include yellowing and strength reduction. In Fig. 8, the whiteness of both alka-

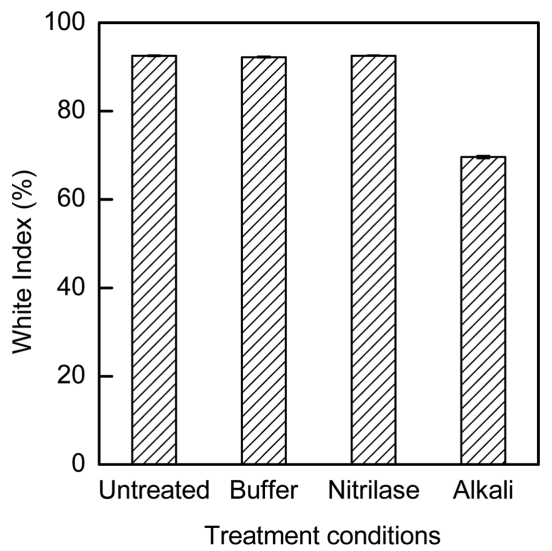

Fig. 8. White index of acrylic fabrics after various treatment conditions. Buffer: buffer treatment at $\mathrm{pH} 8.0,40^{\circ} \mathrm{C}, 5$-h treatment time; Nitrilase: nitrilase treatment at nitrile $10 \%$ (owf), $\mathrm{pH} 8.0,40^{\circ} \mathrm{C}, 5$-h treatment time. Alkali: alkaline treatment with $1 \mathrm{~N} \mathrm{NaOH}, 90^{\circ} \mathrm{C}, 3-\mathrm{h}$ treatment time.

line- and nitrilase-treated acrylic fabrics are compared. The whiteness of the nitrilase-treated sample did not change relative to the whiteness of the untreated sample. However, the whiteness of the alkaline-treated acrylic fabrics decreased. ANOVA of the whiteness confirmed the significance levels; in particular, $F=$ 1879.901 and $p=.000$ at $95 \%$. The Duncan analysis indicated that the fabrics could be divided into the following two groups based on the whiteness: (1) Untreated, buffer-treated, and nitrilase-treated samples and (2) alkaline-treated samples. Thus, ANOVA and Duncan analyses demonstrated that alkaline hydrolysis significantly decreased the whiteness and the nitrilase-treated acrylic fabric had a whiteness similar to that of the untreated sample.

Fig. 9 shows the weight loss and tensile strength loss of the fabrics after treatments under various conditions. The tensile strength of the nitrilase-treated acrylic fabrics remained unchanged after

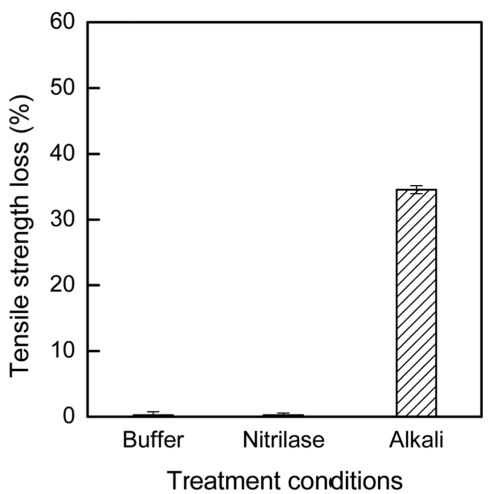

(b)

Fig. 9. Weight loss (a) and tensile strength loss (b) of acrylic fabrics after various treatment conditions. Buffer: buffer treatment at pH 8.0 , $40^{\circ} \mathrm{C}, 5$ h treatment time; Nitrilase: nitrilase treatment at $10 \%$ (owf) nitrile, $\mathrm{pH} 8.0,40^{\circ} \mathrm{C}, 5$-h treatment time. Alkali: alkaline treatment with $1 \mathrm{~N} \mathrm{NaOH}, 90^{\circ} \mathrm{C}$, 3-h treatment time. 


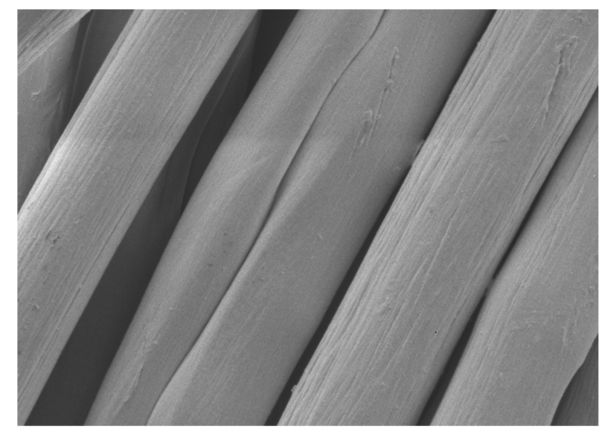

(a) Untreated

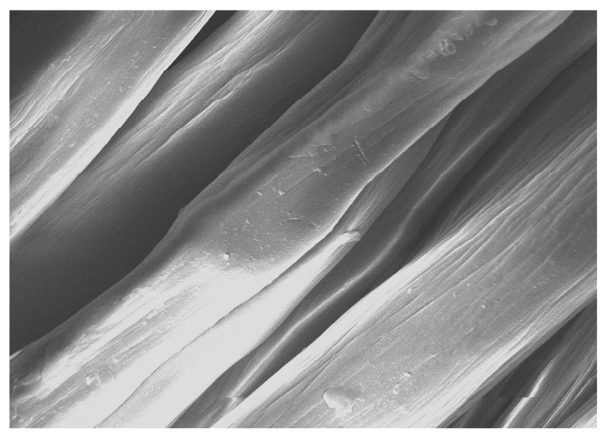

(b) Nitrilase

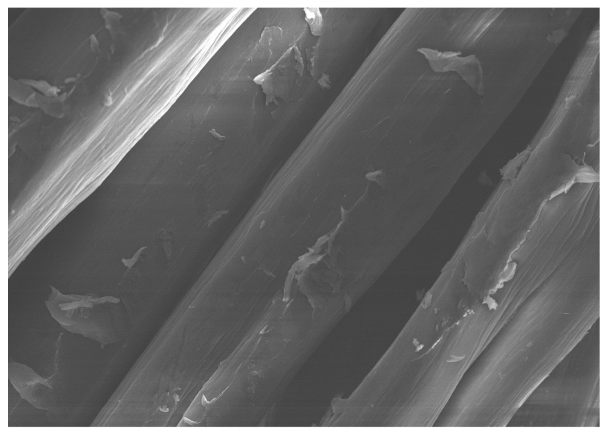

(c) Alkali

Fig. 10. SEM micrographs of acrylic fabrics after various treatment conditions $(2000 \times)$. (a) Untreated: untreated acrylic fabric. (b) Nitrilase: nitrilase treatment at $10 \%$ (owf) nitrile, $\mathrm{pH} 8.0,40^{\circ} \mathrm{C}, 5$-h treatment time. (c) Alkali: alkali treatment with $1 \mathrm{~N} \mathrm{NaOH}, 90^{\circ} \mathrm{C}$, 3-h treatment time.

nitrilase hydrolysis. Additionally, the weight loss caused by nitrilase treatment was only approximately $0.48 \%$. The major advantage of enzymatic modifications of a synthetic fiber over chemical modifications is the highly specific, non-destructive transformation of the targeted surfaces (Gübitz \& Cavaco-Paulo, 2003). Thus, nitrilase hydrolysis is the preferred method for modifying the surfaces of synthetic fibers, and the tensile strength of the fabric was maintained after the treatment. However, the strength loss of the alkaline-hydrolyzed acrylic fabrics was presented approximately $35 \%$ compared with that of the untreated fabric.

Fig. 10 shows the surface morphology of acrylic fabrics after various treatment conditions. In the SEM micrographs, the untreated and nitrilase-treated acrylic fabrics do not show any difference. Thus, nitrilase hydrolysis influenced the conversion of nitriles into carboxylate ions and ammonia on the fiber surfaces without causing any mechanical change. However, with alkaline hydrolysis, the surface roughness was more pronounced. The fiber surfaces peeled off partly and formed fibrils.

Therefore, it was proven that nitrilase hydrolysis improved the sensitivity of acrylic fabrics to basic dye similarly to alkaline hydrolysis without the drawbacks of yellowing and decreased strength caused by alkaline hydrolysis. Thus, the nitrilase treatment of acrylic fabrics could be used as a substitute for alkaline hydrolysis. The nitrilase treatment is also advantageous because it can be performed under conditions milder than those used in alkaline hydrolysis.

\section{Conclusion}

This study determined the optimum conditions for the hydrolysis of acrylic woven fabrics using a commercial nitrilase. To optimize the nitrilase hydrolysis conditions, the hydrolytic activity of nitrilase was evaluated from the amount of ammonia released into the treatment liquid. The hydrophilic ionic groups formed on the surface of the acrylic fabric were evaluated by XPS, as well as WCA and water absorption time. The sensitivities to basic dye, whiteness, strengths, and weight losses of nitrilase- and alkalinehydrolyzed fabrics were compared.

On the basis of the amount of ammonia released into the treatment liquid by nitrilase hydrolysis, the optimum conditions were determined to be $\mathrm{pH} 8.0$ at $40^{\circ} \mathrm{C}$ for a treatment time of $5 \mathrm{~h}$. The XPS analysis indicated the amounts of elements affected by nitrilase hydrolysis. The carbon content of the nitrilase-treated samples decreased slightly in response to the increase in the oxygen content. These results indicate that the nitrile groups were converted into carboxylate ions. The surface of the acrylic fabric became less hydrophobic after nitrilase hydrolysis and the wettability improved.

The nitrilase hydrolysis improved the sensitivity of acrylic fabrics to basic dye similarly to alkaline hydrolysis without the drawbacks of yellowing and decreased strength caused by alkaline hydrolysis. Therefore, the present study proved the possibility of substituting alkaline hydrolysis with enzymatic hydrolysis on acrylic woven fabrics using a commercial nitrilase.

\section{Acknowledgement}

This Research was supported by the Sookmyung Women's Uni- 
versity Research Grants (1-1503-0057).

\section{References}

Babu, V., Shilpi, Gupta, M., \& Choudhury, B. (2011). Opportunities and challenges for enzymatic surface modification of synthetic polymers. In S. K. Sharma \& A. Mudhoo (Eds.), Green chemistry for environmental sustainability (pp. 93-104). New York, NY: CRC Press.

Banerjee, A., Sharma, R., \& Banerjee, U. C. (2003). A rapid and sensitive fluorometric assay method for the determination of nitrilase activity. Biotechnology and Applied Biochemistry, 37(3), 289-293. doi:10.1042/BA20020106

Battistel, E., Morra, M., \& Marinetti, M. (2001). Enzymatic surface modification of acrylonitrile fibers. Applied Surface Science, 177 (1-2), 32-41. doi:10.1016/S0169-4332(01)00193-3

Blyth, R. I. R., Buqa, H., Netzer, F. P., Ramseya, M. G., Besenhard, J. O., Golobic, P., \& Winterb, M. (2000). XPS studies of graphite electrode materials for lithium ion batteries. Applied Surface Science, 167(1-2), 99-106. doi:10.1016/S0169-4332(00)00525-0

Bornscheuer, U. T., \& Kazlauskas, R. J. (2005). Hydrolases in organic synthesis: Regio- and stereoselective biotransformations ( $2^{\text {nd }}$ ed.). (pp. 227-240). Manhattan: John Wiley @ Sons.

Fischer-Colbrie, G., Matama, T., Heumann, S., Martinkova, L., Paulo, A. C., \& Guebitz, G. (2007). Surface hydrolysis of polyacrylonitrile with nitrile hydrolyzing enzymes from micrococcus luteus BST20. Journal of Biotechnology, 129(1), 62-68. doi:10.1016/ j.jbiotec.2006.11.018

Gong, J. S., Lu, Z. M., Li, H., Shi, J. S., Zhou, Z. M., \& Xu, Z. H. (2012). Nitrilases in nitrile biocatalysis: Recent progress and forthcoming research. Microbial Cell Factories, 11(1), 142-159. doi: 10.1186/1475-2859-11-142

Gübitz, G. M., \& Cavaco-Paulo, A. (2003). New substrates for reliable enzymes: Enzymatic modification of polymers. Current Opinion in Biotechnology, 14(6), 577-582. doi:10.1016/j.copbio.2003.09.010

Gupta, M. L., Gupta, B., Oppermann, W., \& Hartmann, G. (2004). Surface modification of polyacrylonitrile staple fibers via alkaline hydrolysis for superabsorbent applications. Journal of Applied Polymer Science, 91(5), 3127-3133. doi:10.1002/app.13486

Kim, H. R., \& Song, W. S. (2010). Lipase treatment to improve hydrophilicity of polyester fabrics. International Journal of Clothing Science and Technology, 22(1), 25-34. doi:10.1108/09556221011008785

Kim, S. H., Lee, T. S., \& Park, W. H. (2002). Preparation of antimicrobial fibers through chemical modification of acrylic fibers. Textile Science and Engineering, 39(4), 390-395.

Kobayashi, M., \& Shimizu, S. (2000). Nitrile hydrolases. Current Opinion in Chemical Biology, 4(1), 95-102.

Majumdar, A., Das, S. C., Shripathi, T., \& Hippler, R. (2012). Chemical synthesis and surface morphology of amorphous hydrogenated carbon nitride film deposited by $\mathrm{N}_{2} / \mathrm{CH}_{4}$ dielectric barrier discharge plasma. Composite Interfaces, 19(3-4), 161-170. doi:10.1080/ 15685543.2012.699751

Matamá, T., Carneiro, F., Caparrós, C., Gübitz, G. M., \& CavacoPaulo, A. (2007). Using a nitrilase for the surface modification of acrylic fibres. Biotechnology Journal, 2(2), 353-360. doi:10.1002/ biot. 200600068

Tauber, M. M., Cavaco-Paulo, A., Robra, K. H., \& Gübitz, G. M. (2000). Nitrile hydratase and amidase from rhodococcus rhodochrous hydrolyze acrylic fibers and granular polyacrylonitriles. Applied and Environmental Microbiology, 66(4), 1634-1638. doi:10.1128/AEM.66.4.1634-1638.2000

Wang, N., Xu, Y., \& Lu, D. N. (2004). Enzymatic surface modification of acrylic fiber. AATCC Review, 4(9), 28-30.

(Received 7 November 2016; 1st Revised 7 December 2016; 2nd Revised 20 December 2016; Accepted 23 December 2016) 